\title{
Differentially co-expressed interacting protein pairs discriminate samples under distinct stages of HIV type 1 infection
}

\author{
Dukyong Yoon ${ }^{1 \dagger}$, Hyosil Kim ${ }^{1 \dagger}$, Haeyoung Suh-Kim² ${ }^{2}$ Rae Woong Park ${ }^{1 *}$, KiYoung Lee ${ }^{1,3^{*}}$
}

From 22nd International Conference on Genome Informatics

Busan, Korea. 5-7 December 2011

\begin{abstract}
Background: Microarray analyses based on differentially expressed genes (DEGs) have been widely used to distinguish samples across different cellular conditions. However, studies based on DEGs have not been able to clearly determine significant differences between samples of pathophysiologically similar HIV-1 stages, e.g., between acute and chronic progressive (or AIDS) or between uninfected and clinically latent stages. We here suggest a novel approach to allow such discrimination based on stage-specific genetic features of HIV-1 infection. Our approach is based on co-expression changes of genes known to interact. The method can identify a genetic signature for a single sample as contrasted with existing protein-protein-based analyses with correlational designs.
\end{abstract}

Methods: Our approach distinguishes each sample using differentially co-expressed interacting protein pairs (DEPs) based on co-expression scores of individual interacting pairs within a sample. The co-expression score has positive value if two genes in a sample are simultaneously up-regulated or down-regulated. And the score has higher absolute value if expression-changing ratios are similar between the two genes. We compared characteristics of DEPs with that of DEGs by evaluating their usefulness in separation of HIV-1 stage. And we identified DEP-based network-modules and their gene-ontology enrichment to find out the HIV-1 stage-specific gene signature.

Results: Based on the DEP approach, we observed clear separation among samples from distinct HIV-1 stages using clustering and principal component analyses. Moreover, the discrimination power of DEPs on the samples (70-100\% accuracy) was much higher than that of DEGs (35-45\%) using several well-known classifiers. DEP-based network analysis also revealed the HIV-1 stage-specific network modules; the main biological processes were related to "translation," "RNA splicing," "mRNA, RNA, and nucleic acid transport," and "DNA metabolism." Through the HIV-1 stage-related modules, changing stage-specific patterns of protein interactions could be observed.

Conclusions: DEP-based method discriminated the HIV-1 infection stages clearly, and revealed a HIV-1 stagespecific gene signature. The proposed DEP-based method might complement existing DEG-based approaches in various microarray expression analyses.

\footnotetext{
* Correspondence: veritas@ajou.ac.kr; kiylee@ajou.ac.kr

+ Contributed equally

'Department of Biomedical Informatics, Ajou University School of Medicine,

Suwon 443-749, Korea

Full list of author information is available at the end of the article
} 


\section{Background}

Human immunodeficiency virus type 1 (HIV-1) has been demonstrated to damage the human immune system, finally leading to acquired immunodeficiency syndrome (AIDS), which is characterized by vulnerability to life-threatening opportunistic infections. The natural progression of HIV-1 consists of the acute stage, the clinical latency stage, and AIDS [1]. The acute stage (Acute), the first stage of HIV-1 infection, results from contamination with the HIV-1 virus through body fluids such as blood, semen, or vaginal fluid. In this stage, the copy number of HIV-1 virus rapidly increases, and the number of $\mathrm{CD} 4+\mathrm{T}$ cells markedly decreases [2]. However, most patients with HIV-1 infection recover from the acute stage without treatment within 3 to 6 weeks and have a period of clinical latency of 8 to 10 years [1]. Although there are no clinical manifestations and the CD4+ T-cell count is almost recovered during the clinical latency stage, it has been reported that immune damage persistently occurs [3]. Among the HIV-infected population, approximately 5 to $8 \%$ of patients remain clinically stable for decades. They have been referred to as long-term non-progressors (Non-progressive) [4]. However, most patients undergo chronic progressive infection (Chronic) that finally leads to AIDS, at which point the CD4+ T-cell count drops below 200 cells $/ \mu \mathrm{L}$, and $\mathrm{T}$ cell-mediated immunity fails to protect the body from pathogens.

Several studies have attempted to reveal the mechanism of HIV-1 pathogenesis at the genomic level using microarray experiments. Using analysis of differentially expressed genes (DEGs) across HIV-1 infection stages, Hyrcza et al. found that expression of interferon-stimulated genes is increased in the early and chronic progressive stages [5]. Li et al., by a similar DEG-based analysis using lymphatic tissue microarrays, showed that each stage has relatively different gene expression patterns [6]. These studies have enhanced our knowledge about the pathogenic mechanism of HIV-1. One of the common limits of these studies, however, is that DEGbased expression analysis cannot identify an HIV-1 stage-specific gene signature that can clearly discriminate pathophysiologically similar stages, such as between Acute and Chronic stages or between Uninfected and Non-progressive stages [5,6].

Recently, protein-interaction-based analyses with correlational designs have been successfully applied to discover a discriminant genetic signature for a specific condition, but not for an individual sample, using microarray analysis $[7,8]$. These analyses usually have different assigned weights for an interacting protein pair based on degrees of correlation of expression levels under specific conditions. Genes or gene products do not work alone, but rather function in relationship with other genes or proteins in a real molecular setting [9]. Moreover, the degree of correlation between members of an interacting protein pair under a specific condition might provide evidence for the degree of functional relationship under that condition. However, this approach requires multiple samples under a target condition to extract the genetic features for the condition; thus, it cannot be used for a genetic signature of a single sample, which is required to validate or test whether a new sample has a signature similar to those of other samples in a certain group.

Here, we suggest a novel protein-interaction-based method to capture a genetic signature for a single sample under a specific condition. To achieve this purpose, we assigned a co-expression (or co-changing) score to a protein-protein interaction by comparing the expression-change ratios of the two genes in a sample with representative values. After assigning co-expression scores for each sample, we found differentially coexpressed interacting protein pairs (DEPs) among conditions for a condition-specific signature. We applied the DEP-based method to samples representing the clinical stages of HIV-1 infection to discover an HIV-1 stagespecific signature.

\section{Methods}

\section{Acquisition of HIV-1-infected gene expressions and} human protein-protein interactions

For HIV-1 expression data, we downloaded the Series GSE6740 dataset from the GEO database (http://www. ncbi.nlm.nih.gov/geo). The dataset contains stage-specific gene expressions of CD4+ and CD8+ cells from a cohort of HIV-infected individuals [5]. The HIV-infected individuals had not been treated at the time samples were obtained. The profiles of CD4+ and CD8+ T cells from individuals with early HIV-1 infections (Acute), non-progressive HIV-1 infections with low or undetectable viral loads (Non-progressive), chronic progressive HIV-1 infections (Chronic), and uninfected individuals (Uninfected) were selected (Figure 1A). The expression profiles were normalized using a quantile normalization method implemented in MATLAB R2009b (Mathworks, Natick, MA, USA) (Figure 1B). Final expression datasets contained 10 Acute, 10 Non-progressive, 10 Chronic samples, and 10 Uninfected samples. For human protein-protein interactions (PPIs), we used the data of Lee et al. [10], which incorporated public databases such as DIP [11], BIND [12], HPRD [13], and REACTOME [14]. The data set also included the results of several recent genomewide studies [15-18]. A total of 80970 interactions among 10819 human proteins were prepared. For the subsequent analyses, including calculating co-expression 


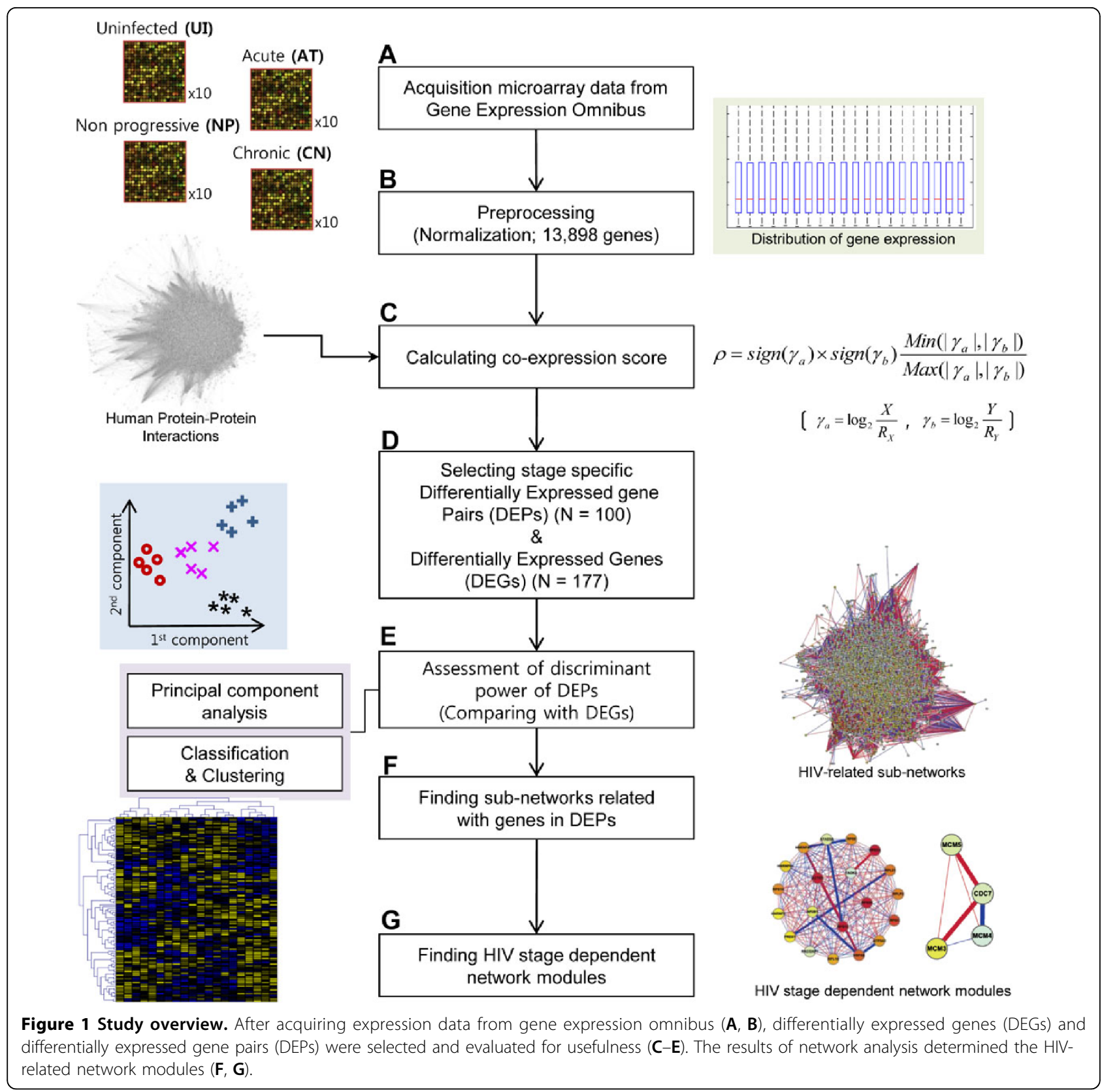

scores and identifying differentially co-expressed interacting protein pairs, only these interactions were considered.

\section{Calculating a co-expression score between two interacting gene products}

A co-expression (or co-changing) score between two interacting gene products in a sample was calculated by following two steps (Figure 1C). First, expression-changing ratios $(\gamma)$ of two genes, $a$ and $b$, were defined as follows:

$$
\gamma_{a}=\log _{2} \frac{X}{R_{X}} \text { and } \gamma_{b}=\log _{2} \frac{Y}{R_{Y}}
$$

where $X$ (or $Y$ ) indicates the normalized expression level of gene $a$ (or $b$ ), and $R_{X}$ (or $R_{Y}$ ) is a representative expression level of gene $a$ (or $b$ ). In this study, the median value of expressions across samples was used as a representative value of a specific gene. Next, a coexpression score $(\rho)$ between the two interacting gene products in the sample was calculated using the following equation: 


$$
\rho(a, b)=\operatorname{sign}\left(\gamma_{a}\right) \times \operatorname{sign}\left(\gamma_{b}\right) \frac{\operatorname{Min}\left(\left|\gamma_{a}\right|,\left|\gamma_{b}\right|\right)}{\operatorname{Max}\left(\left|\gamma_{a}\right|,\left|\gamma_{b}\right|\right)}
$$

where $\operatorname{Min}(p, q)$ or $\operatorname{Max}(p, q)$ indicates the minimum or maximum value between $p$ and $q$, respectively, and $\operatorname{sign}(x)$ indicates the sign of $x$. Note that the coexpression score $\rho$ has a positive value if $X$ and $Y$ are simultaneously greater (or smaller) than $R_{X}$ and $R_{Y}$; otherwise, it has a negative value. Moreover, the coexpression score $\rho$ has a higher absolute value if the absolute values of expression-changing ratios are similar between the two genes.

\section{Identifying DEPs and DEGs}

To identify DEPs for HIV-1 infection, analysis of variance (ANOVA) and geometric means of differences between median co-expression scores across individual stages were used (Figure 1D). An interaction with a high value of $-\log _{10}(\mathrm{p}$-value of an ANOVA test $) \times($ geometric mean of differences of median co-expression scores) was considered significant, and interactions with higher degrees of significance than a specific cutoff value were selected as DEPs. A similar process was applied to select DEGs, except that expression levels, not co-expression scores, were used. To ensure a balanced comparison, the most highly significant DEGs were selected in a quantity equal to the number of genes. Here, several values from 0.5 to 1 were examined to identify the optimal cutoff value that provided the smallest number of DEPs and DEGs with the best accuracy.

\section{PCA, clustering, and classification analyses of DEPs and DEGs}

To estimate how DEPs reveal HIV-1 stage-specific characteristics, principal-component analysis (PCA) and several well-known classification and clustering methods were used (Figure 1E). PCA transforms attribute values into new ones to create the linear projection of the data that accounts for the most variance in a low-dimensional subspace. Therefore, it provides snapshots of data that we can see at a glance. Global views of DEPs were compared with those of whole genes and DEGs using PCA. PCA was performed using the algorithm implemented in MATLAB, R2009b (MathWorks, Natick, MA, USA).

For clustering, we used hierarchical clustering (HCL) with the K-means methods implemented in MEV4.0 (Multiple Experiment Viewer, http://www.tm4.org) [19]. The HCL method groups samples according to the degree of similarity between them based on feature information (here, DEPs or DEGs) without considering the class information (here, the HIV-1 stages).
Therefore, it was possible to confirm whether the selected feature information of samples (i.e., DEPs or DEGs) is valuable for clustering samples according to stage. K-means clustering, like HCL, is an unsupervised learning method. However, K-means clustering was used to partition DEPs (or DEGs) into some number of clusters. Here, DEPs were clustered into six groups in each cell type (CD4+ and CD8+ cells). Stage-specific clusters in both CD4+ and CD8+ cells were then further characterized through GO term analysis.

For classification methods, we used the J48 decision tree, the SMO support-vector machine, and the multilayer perception artificial neural network, which were implemented in WEKA, version 3.6.3 [20]. Leave-oneout cross-validation (LOOCV) using these methods was applied to estimate the classification performance of selected DEPs (or DEGs) for predicting the disease stages of HIV-1. LOOCV is one of the most popular validation methods; it trains prediction models using all samples except one and then tests the models with the remaining sample. This step then passes through as many iterations as there are samples [21]. For performance measures, we used accuracy, sensitivity, and specificity from a confusion matrix.

\section{DEP-based network-module identification}

To discover HIV-related interaction sub-networks, the prepared PPIs and the list of genes in DEPs were imported into Cytoscape (http://www.Cytoscape.org) [22] with the median co-expression score for each stage (Figure 1F). Next, we included the genes that directly interacted with the genes in DEPs. Using the extended network, MCODE was used to find sets of genes located at the area of dense DEPs (Figure 1G). MCODE is a Cytoscape plug-in and is one of the most popular methods by which to find highly interconnected regions in a network [23]. The score of a sub-network was calculated based on the complexity and density of the network. The top 10 modules with the highest network scores were considered significant since modules with higher network scores showed higher prediction accuracies in previous study [23]. Among 10 significant modules, five modules containing DEPs were finally selected because none of the other five modules included any DEP. Note that a DEP in each sample has its own co-expression score. To find a representative HIV-related module under a specific stage, thus, we used a median value of multiple co-expression scores for an interaction and a median expression level for a protein, respectively.

\section{Gene-ontology enrichment analysis}

For the gene list in DEPs and DEGs, a functional annotation tool called the Database for Annotation, Visualization and Integrated Discovery (DAVID) [24] was applied to find 
functionally enriched terms. DAVID uses a Fisher's exact test to determine whether the proportion of selected genes falling into each category differs from the baseline (here, all genes of Homo sapiens). For selected modules, BiNGO (the Biological Networks Gene-ontology tool) [25] was also used to conduct gene-ontology (GO) enrichment analysis. BiNGO, which is implemented as a plug-in for Cytoscape, maps the predominant functional themes of a given gene set on the GO hierarchy and outputs this mapping as a Cytoscape graph. Hypergeometric distribution was adopted to find a functional degree of overrepresentation of an HIV-related module using this method.

\section{Results}

\section{Identifying DEPs across HIV-1 stages}

We prepared 10 Acute, 10 Non-progressive, 10 Chronic samples, and 10 Uninfected samples of CD4+ and CD8+ cells from a cohort of HIV-infected individuals (Methods). For human protein-protein interactions, we used the data of Lee et al. [10], which cover 80970 interactions among 10819 proteins. To identify DEPs, we first calculated a coexpression score of each interaction using expressionchanging ratios of the corresponding two proteins. After that, we found significantly different pairs using analysis of variance (ANOVA) and geometric means of differences of median levels across HIV-1 stages. Among 80970 interacting protein pairs, 100 pairs were selected as significant DEPs which consist of 177 proteins across HIV-1 infection (Table 1). A DEG-based analysis, on the other hand, selected a different set of 177 genes: only eight genes (or their corresponding encoding proteins) were common to DEPs and DEGs. Figures 2A and 2B show some cases of the selected DEPs in which DEPs could differentiate between pathophysiologically similar HIV-1 stages. For example, the co-expression pattern, illustrated by the line in the figure, between HNRNPM (heterogeneous nuclear ribonucleoprotein $\mathrm{M}$, known to influence pre-mRNA processing and other aspects of mRNA metabolism and transport) and DHX9 (DEAH (Asp-Glu-Ala-His) box polypeptide 9 , known to be involved in the expression and nuclear export of retroviral RNAs and also known to interact with HIV-1 RNA) was positively related to the Acute stage, whereas it was negatively related to Chronic stage (Figure 2A). However, a DEG-based analysis missed the HIV-1 related proteins HNRNPM and DHX9 because of the similar median expression levels across the Acute (black "+") and Chronic (black dashed " $\times$ ") stages. A similar phenomenon was observed between LRRC1 (leucinerich repeat containing 1) and SIAH2 (seven in absentia homolog 2, known to be involved in ubiquitination and proteasome-mediated degradation) which were positive in Uninfected but negative in Non-progressive stages, whereas the median expression levels of LRRC1 and SIAH2 were similar between the two stages (Figure 2B).
The functional characteristics were also significantly different between DEPs and DEGs (Figure 2C). The number of enriched GO terms using the 177 proteins of DEPs was 49 , whereas it was 21 in the case of DEGs (the GO terms with $>10$ genes/proteins and $p$-value of $<1.0 \times 10^{-5}$ using DAVID tools). Among the enriched GO terms, 13 overlapped and were mainly associated with "translation" biological processes. Thirty-six GO terms included only in DEPs were related to responses against endogenous or exogenous stimuli (from transcription to mRNA processing). They were particularly associated with apoptosis ("positive regulation of apoptosis", "positive regulation of programmed cell death", and "positive regulation of cell death"), which is known to be an important factor in the progression of HIV by the resulting depletion of $\mathrm{T}$ helper cells [26]. On the other hand, the GO terms only for DEGs included "response to virus" and "immune response".

\section{PCA results of DEPs and DEGs of HIV-1}

We applied PCA to evaluate the geometric view of the samples in various HIV-1 stages with $i$ ) whole genes of the microarray, $i i)$ the identified DEGs, or iii) the identified DEPs (Figure 3). In the case of whole genes (Figure 3A), the four areas of samples in distinct HIV-1 stages highly overlapped one another with regard to the first three principal components, even though the samples were separable according to cell type (CD4+ cells in red and CD8+ cells in blue). In the PCA analysis with DEGs (Figure 3B), the HIV-1 stages were still not separable; in particular, the Uninfected and Non-progressive areas and the Acute and Chronic areas were highly overlapped. When the identified DEPs were used (Figure 3C), however, all four stages were clearly separable using the three components, regardless of cell type. Interestingly, pathophysiologically similar HIV-1 stages, such as Acute and Chronic or Uninfected and Nonprogressive, were highly discriminable using the first two principal components (middle panel of Figure 3C), although there was an area that overlapped between Chronic and Non-progressive. These four stages were clearly separable both in the first and third planes and in the second and third planes (first and third panels of Figure $3 \mathrm{C}$, respectively). This tendency was also observed in individual cell types, i.e., in CD4+ cells and CD8+ cells. The DEP PCA results showed the most clearly discriminated distribution of the samples across the HIV-1 stages compared with those of whole genes or DEGs regardless of cell type.

\section{Clustering results of DEPs in HIV-1}

We applied clustering methods to the samples without seeing their HIV-1 stage information. When the extracted DEG-based features were used for the individual CD4+ samples (Figure 4A), the samples were 
Table 1 Protein pairs included in the top-30 DEPs

\begin{tabular}{|c|c|c|c|c|c|c|}
\hline Protein 1 & Name of Protein 1 & Protein 2 & Name of Protein 2 & P-value & G-mean & Significance \\
\hline NUTF2 & nuclear transport factor 2 & NUP62 & nucleoporin $62 \mathrm{kDa}$ & $7.5 \mathrm{E}-06$ & 0.37 & 1.90 \\
\hline CDC7 & cell division cycle 7 homolog (S. cerevisiae) & MCM3 & $\begin{array}{l}\text { minichromosome maintenance complex } \\
\text { component } 3\end{array}$ & $1.3 \mathrm{E}-05$ & 0.34 & 1.66 \\
\hline VAMP1 & $\begin{array}{l}\text { vesicle-associated membrane protein } 1 \\
\text { (synaptobrevin 1) }\end{array}$ & ARFGAP1 & $\begin{array}{l}\text { ADP-ribosylation factor GTPase activating } \\
\text { protein } 1\end{array}$ & 4.3E-05 & 0.36 & 1.56 \\
\hline HSPA8 & heat shock $70 \mathrm{kDa}$ protein 8 & TADA3L & transcriptional adaptor 3 & $1.0 \mathrm{E}-05$ & 0.26 & 1.29 \\
\hline TNR & tenascin R (restrictin, janusin) & NFASC & neurofascin & $1.6 \mathrm{E}-04$ & 0.33 & 1.2 \\
\hline ARHGEF2 & $\begin{array}{l}\text { Rho/Rac guanine nucleotide exchange } \\
\text { factor (GEF) } 2\end{array}$ & PRKCl & protein kinase $C$, iota & $6.4 \mathrm{E}-05$ & 0.28 & 1.17 \\
\hline PDGFRB & $\begin{array}{l}\text { platelet-derived growth factor receptor, } \\
\text { beta polypeptide }\end{array}$ & SNX2 & sorting nexin 2 & 8.7E-05 & 0.28 & 1. \\
\hline RFC5 & replication factor C (activator 1) 5, 36.5kDa & POLA1 & $\begin{array}{l}\text { polymerase (DNA directed), alpha 1, } \\
\text { catalytic subunit }\end{array}$ & $5.5 \mathrm{E}-05$ & 0.27 & 1.13 \\
\hline NFIB & nuclear factor $\mathrm{I} / \mathrm{B}$ & RFX1 & $\begin{array}{l}\text { regulatory factor } X, 1 \text { (influences HLA class } \\
\text { II expression) }\end{array}$ & $3.4 \mathrm{E}-04$ & 0.32 & 1. \\
\hline EIF3| & $\begin{array}{l}\text { eukaryotic translation initiation factor } 3 \text {, } \\
\text { subunit I }\end{array}$ & SUMO4 & $\begin{array}{l}\text { SMT3 suppressor of mif two } 3 \text { homolog } 4 \\
\text { (S. cerevisiae) }\end{array}$ & 1.7E-05 & 0.23 & 1.09 \\
\hline COL17A1 & collagen, type XVII, alpha 1 & LAD1 & ladinin 1 & $3.9 \mathrm{E}-04$ & 0.31 & 1.07 \\
\hline IRS1 & insulin receptor substrate 1 & UBTF & $\begin{array}{l}\text { upstream binding transcription factor, RNA } \\
\text { polymerase I }\end{array}$ & $4.5 \mathrm{E}-04$ & 0.32 & 1.05 \\
\hline CAV1 & caveolin 1, caveolae protein, $22 \mathrm{kDa}$ & TRAF6 & TNF receptor-associated factor 6 & $1.0 \mathrm{E}-04$ & 0.26 & 1.05 \\
\hline RPS14 & ribosomal protein S14 & RPS27A & ribosomal protein S27a & $3.2 \mathrm{E}-03$ & 0.41 & 1.03 \\
\hline HNRNPA2B1 & $\begin{array}{l}\text { heterogeneous nuclear ribonucleoprotein } \\
\text { A2/B1 }\end{array}$ & HNRNPH1 & $\begin{array}{l}\text { heterogeneous nuclear ribonucleoprotein } \\
\mathrm{H} 1(\mathrm{H})\end{array}$ & 4.1E-05 & 0.23 & 1.02 \\
\hline TAF4 & $\begin{array}{l}\text { TATA box binding protein (TBP)-associated } \\
\text { factor, } 135 \mathrm{kDa}\end{array}$ & CBX3 & chromobox homolog 3 & $1.0 \mathrm{E}-03$ & 0.34 & 1.01 \\
\hline VPS11 & $\begin{array}{l}\text { vacuolar protein sorting } 11 \text { homolog (S. } \\
\text { cerevisiae) }\end{array}$ & VPS45 & $\begin{array}{l}\text { vacuolar protein sorting } 45 \text { homolog (S. } \\
\text { cerevisiae) }\end{array}$ & $2.4 \mathrm{E}-04$ & 0.28 & 1. \\
\hline ATP5F1 & $\begin{array}{l}\text { ATP synthase, H+ transporting, } \\
\text { mitochondrial Fo complex, subunit B1 }\end{array}$ & ATP5J2 & $\begin{array}{l}\text { ATP synthase, } \mathrm{H}+\text { transporting, } \\
\text { mitochondrial Fo complex, subunit F2 }\end{array}$ & $8.1 \mathrm{E}-05$ & 0.24 & 0.99 \\
\hline POLR2G & $\begin{array}{l}\text { polymerase (RNA) II (DNA directed) } \\
\text { polypeptide G }\end{array}$ & SF3B2 & splicing factor $3 \mathrm{~b}$, subunit $2,145 \mathrm{kDa}$ & $2.6 \mathrm{E}-03$ & 0.38 & 0.9 \\
\hline PDPK1 & $\begin{array}{l}\text { 3-phosphoinositide dependent protein } \\
\text { kinase-1 }\end{array}$ & PRKCQ & protein kinase $\mathrm{C}$, theta & $4.5 \mathrm{E}-05$ & 0.22 & 0. \\
\hline EP300 & E1A binding protein p300 & TF & transferrin & $5.3 \mathrm{E}-04$ & 0.30 & 0.97 \\
\hline RPS5 & ribosomal protein $\mathrm{S} 5$ & RPL28 & ribosomal protein L28 & $1.4 \mathrm{E}-03$ & 0.34 & 0.9 \\
\hline ELK1 & ELK1, member of ETS oncogene family & GRB10 & growth factor receptor-bound protein 10 & $9.7 \mathrm{E}-04$ & 0.32 & 0.96 \\
\hline RPS13 & ribosomal protein S13 & ATAD3A & ATPase family, AAA domain containing $3 \mathrm{~A}$ & $5.8 \mathrm{E}-05$ & 0.23 & 0.95 \\
\hline PABPC1 & poly(A) binding protein, cytoplasmic 1 & RPS4Y1 & ribosomal protein S4, Y-linked 1 & $2.5 \mathrm{E}-03$ & 0.37 & 0.95 \\
\hline PRPF4 & $\begin{array}{l}\text { PRP4 pre-mRNA processing factor } 4 \\
\text { homolog (yeast) }\end{array}$ & PPIH & peptidylprolyl isomerase $\mathrm{H}$ (cyclophilin H) & $6.5 \mathrm{E}-04$ & 0.29 & 0.93 \\
\hline ZFP36 & $\begin{array}{l}\text { zinc finger protein } 36, \mathrm{C} 3 \mathrm{H} \text { type, homolog } \\
\text { (mouse) }\end{array}$ & $\mathrm{EIF} 2 \mathrm{C} 4$ & eukaryotic translation initiation factor $2 \mathrm{C}, 4$ & $6.3 \mathrm{E}-04$ & 0.29 & 0.93 \\
\hline RPLP2 & ribosomal protein, large, P2 & RPL29 & ribosomal protein L29 & $1.2 \mathrm{E}-03$ & 0.32 & 0.93 \\
\hline HSF1 & heat shock transcription factor 1 & STIP1 & stress-induced-phosphoprotein 1 & $1.5 \mathrm{E}-04$ & 0.24 & 0.92 \\
\hline GSK3B & glycogen synthase kinase 3 beta & FUS & fused in sarcoma & 2.7E-03 & 0.35 & 0.9 \\
\hline
\end{tabular}

Here, significance is "-log(P-value) $\times$ G-mean".

mixed together across the different HIV-1 stages, especially between Acute and Chronic (in orange and red, respectively) and between Non-progressive and Uninfected (in blue and green, respectively). This indicates that Chronic samples and Acute samples have similar expression patterns, as do Non-progressive and Uninfected stages. When DEP-based features were used with the same hierarchical clustering method, however, same-stage samples were clustered first (Figure 4B). After grouping samples according to stage information, Chronic and Non-progressive samples were then clustered. Finally, Uninfected and Acute were clustered in sequence. The heat maps of hierarchical clustering results showed that the co-expression patterns of each 


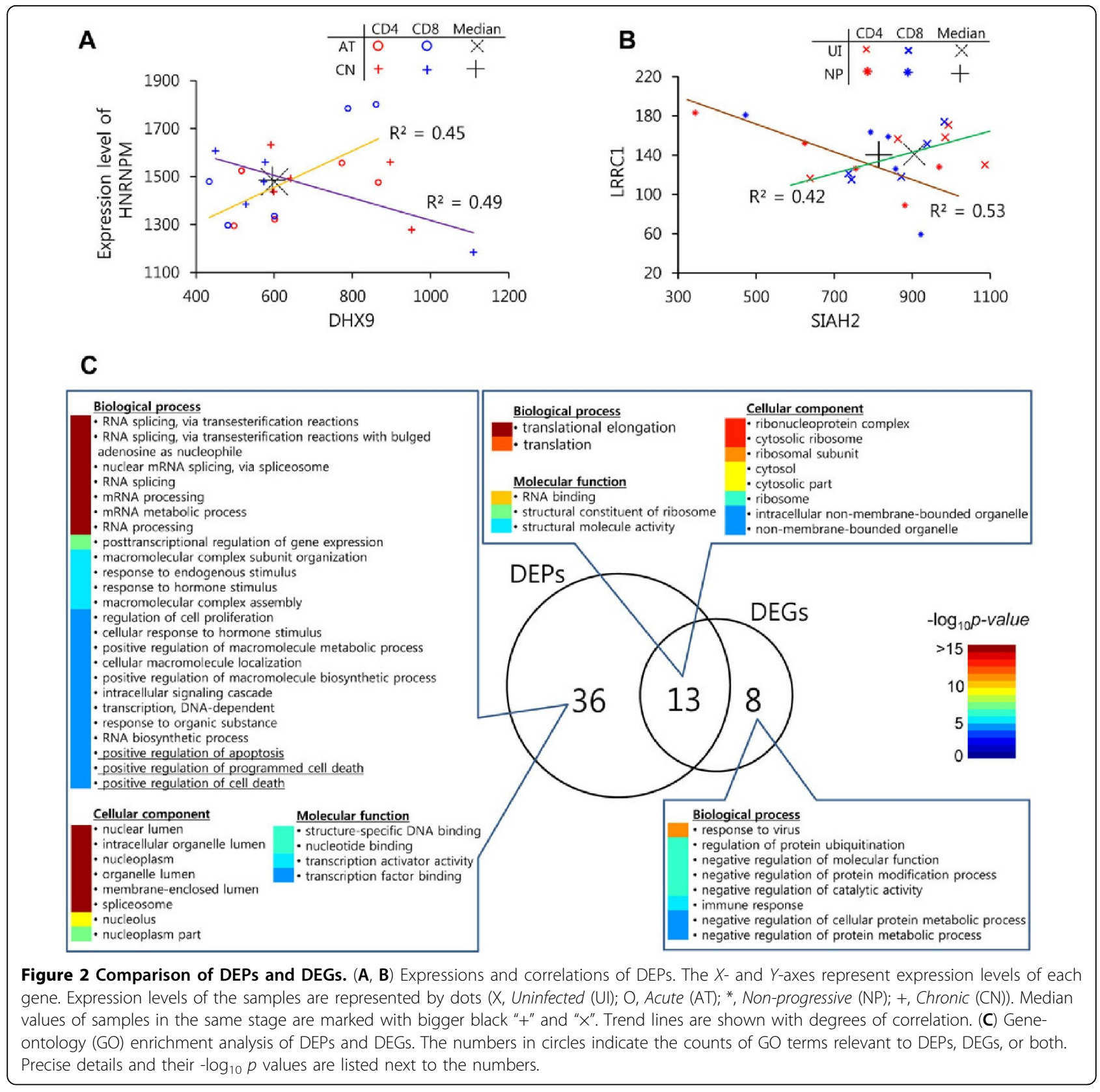

stage were quite different across the HIV-1 stages, whereas individual gene expression patterns lacked distinct patterns according to stage, especially between pathophysiologically similar HIV-1 stages (e.g., between Acute and Chronic or between Uninfected and Non-progressive stages). This tendency was also observed in CD8+ cells. Moreover, the DEP-based features clearly clustered each stage of HIV-1, regardless of cell type. Co-expression patterns of samples were preserved across individual HIV-1 stages.

To discover stage-specific co-expressed pairs, we next clustered the 100 identified DEPs into six groups using a K-means clustering method. Different cell types might be associated with different DEP groups; therefore, we identified separate clusters using only CD4+ samples or only CD8+ samples. Of the six groups, four groups in each cell type showed stage-specific co-expression patterns. Surprisingly, the median co-expression scores of groups across samples were quite similar between CD4+ and CD8+ cells. Moreover, each pair of groups with a similar co-expression pattern shared many DEPs. Using the DEPs shared between CD4+ and CD8+ cells, we analyzed enriched GO terms (Figure 4C). Common biological functions of all four clusters were "RNA splicing" 

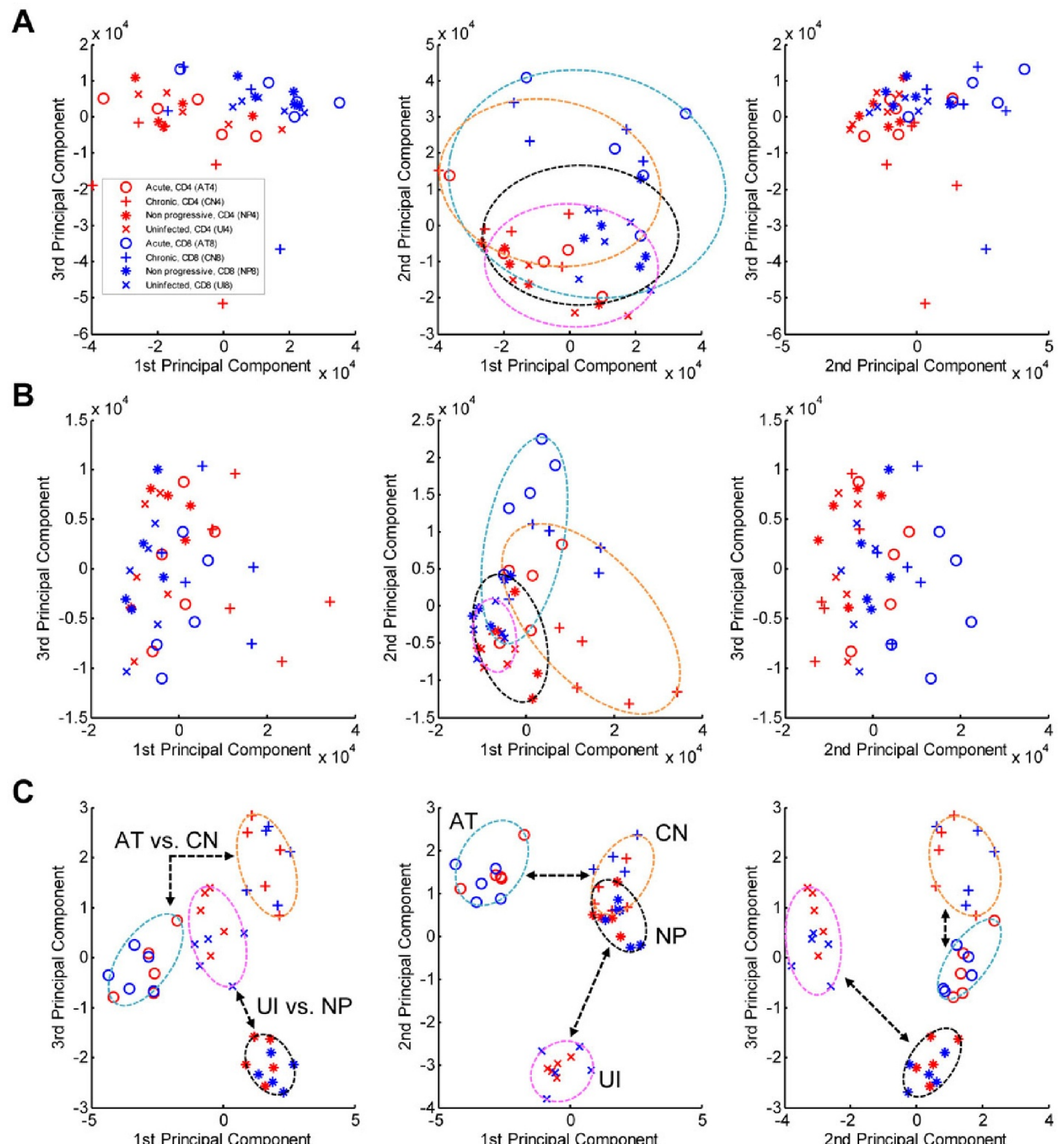

Figure 3 Result of principal-component analysis. (A) Global view of all expression data sets using principal component analysis. The first principal component accounts for as much variability in the expression of total genes as possible. The second and third components account for as much of the remaining variability as possible. Results of principal component analysis with DEGs and DEPs are shown in (B) and (C), respectively.

and "mRNA processing". The first cluster, which was composed of 14 DEPs, had lower co-expression scores only in the Uninfected stage. The genes of the 14 DEPs are known to play a major role in "transcription" in addition to the common functions. In contrast, the second cluster, which had 10 DEPs with lower coexpression scores only in the Acute stage, was involved in "translation" rather than "transcription". The genes in the seven DEPs of the third cluster, which had higher co-expression scores only in the Non-progressive stages, 

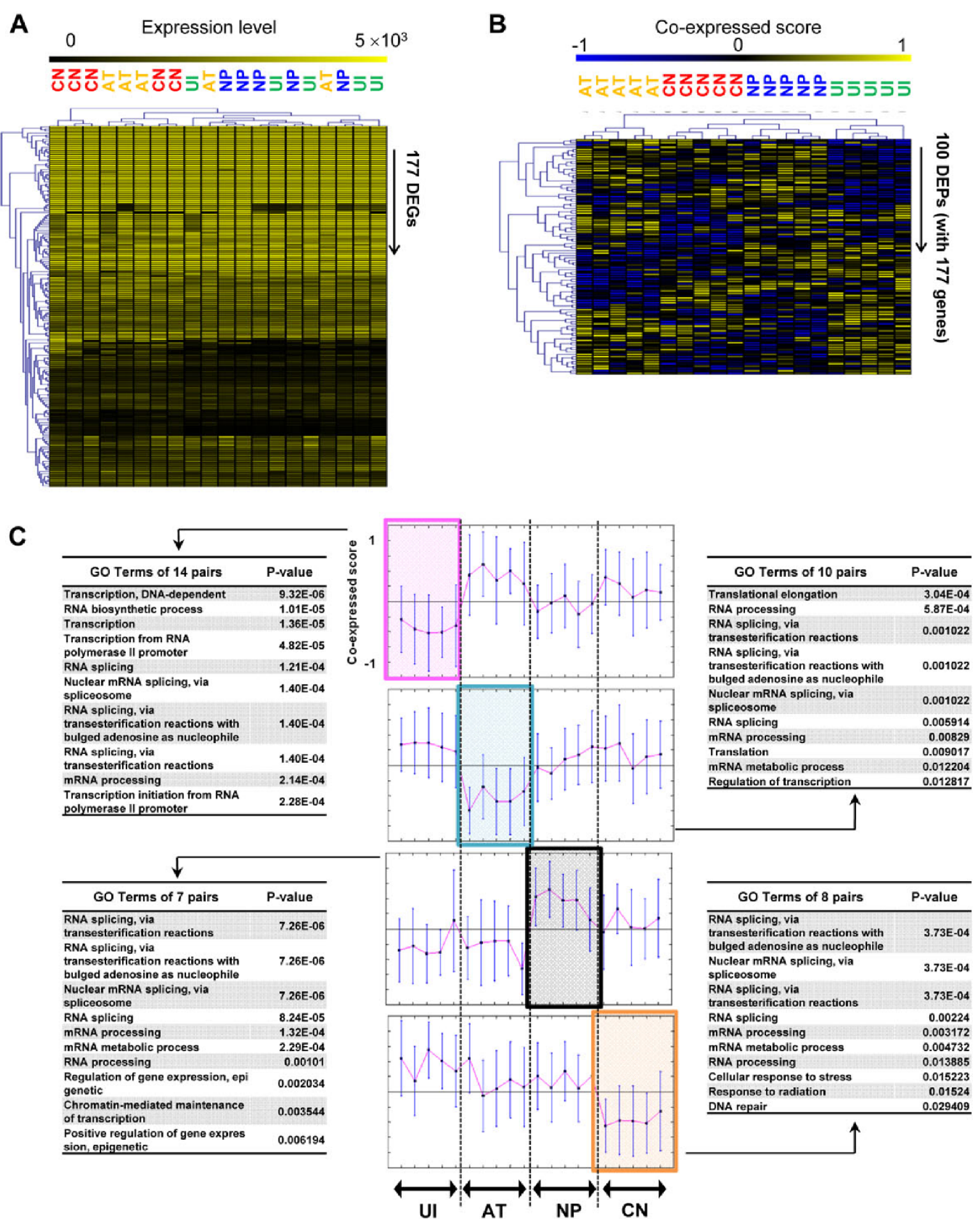

Figure 4 Clustering results using DEPs and DEGs. (A) Heat map for expression of DEGs across the samples. Each lane represents the expression profile of one sample. Result of hierarchical clustering with DEGs is shown at the top of the heat map (UI, Uninfected; AT, Acute; NP, Non-progressive; CN, Chronic). (B) Heat map for co-expressed score of DEPs. (C) Four representative clusters of gene pairs. Of six groups of gene pairs clustered by K-means clustering using Pearson's correlation between pairs and samples, four groups had relatively different co-expression scores compared with the other stages. The $X$-axis represents samples, and the $Y$-axis represents co-expression score. The top $10 \mathrm{GO}$ terms related to biological processes are listed in descending order of $p$-values. 
are also known to play a role in "epigenetic processing". Major functions of the last cluster (Chronic) were related to "repair processes" such as "cellular response to stress", "response to radiation" and "DNA repair".

\section{Discriminant power of DEPs for HIV-1 stages}

To directly investigate the discriminant power of DEPs for the HIV-1 stages, we compared the prediction performance of the identified DEPs with that of the DEGs using several well-known classification methods including a decision tree, a support vector machine, and an artificial neural network (Methods). We here used a LOOCV approach with several performance measures including accuracy, sensitivity, and specificity. As shown in Figure 5A, the prediction accuracies $(95 \%, 100 \%$, and $100 \%$, respectively) of DEPs were much higher than those $(45 \%, 62.5 \%$, and $70 \%$, respectively) of DEGs regardless of the classification method used. The better performance of DEPs was also observed with other measures including sensitivity and specificity (Figure 5B). Moreover, among the misclassification cases, the $71.4 \%$ incorrect prediction (35 among 49 wrong predictions) of the three models built with DEGs was caused by misclassification between Acute and Chronic or between Uninfected and Non-progressive (Figure 5C). In the case of DEPs, however, the three models did not misclassify

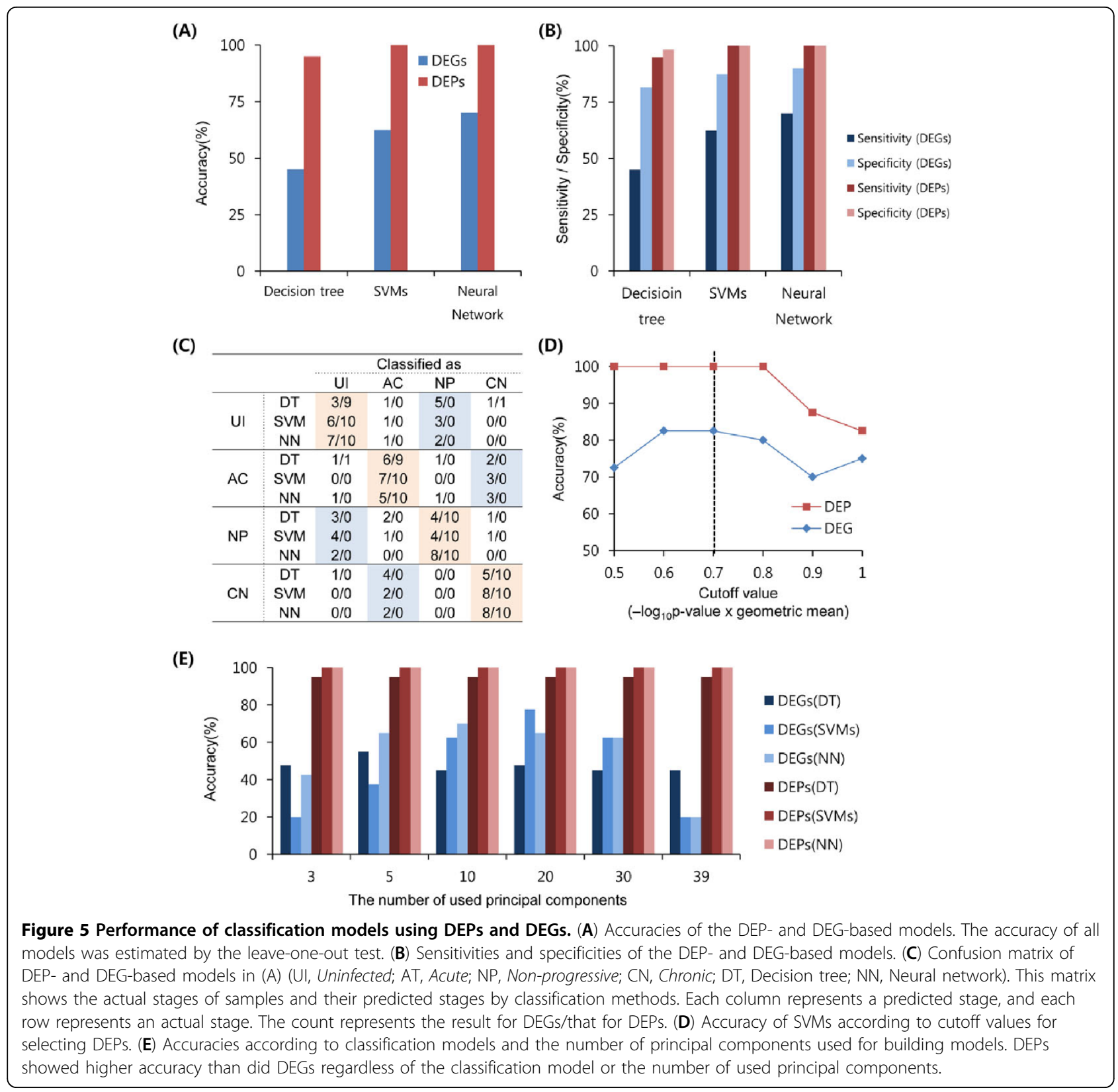


any case between these stages. The only two misclassified cases were misclassification of an Uninfected sample as Chronic and an Acute sample as Uninfected. The DEP-based features correctly classified the pathophysiologically similar HIV-1 stage pairs.

Next, we evaluated the influence of the number of DEPs (or DEGs) on the power for discriminating the HIV-1 stages. The performance was evaluated by a support-vector machine with various cutoff values for the degree of significance of DEPs from 0.5 to 1.0. Here, as many DEGs were selected as there were DEP genes. As shown in Figure 5D, the highest accuracy was achieved at values of 0.6 and 0.7 (100\% accuracy in classifying HIV-1 stages with DEPs and $82.5 \%$ accuracy with DEGs). Thus, in this study, we selected 0.7 as the cutoff value for DEP selection because it revealed the highest level of performance with a smaller number of DEPs and DEGs. As a result, 100 DEPs consisting of 177 genes and 177 DEGs were selected. Additionally, we investigated the impact of the number of selected features from PCA analysis with the selected DEPs and DEGs. In the PCA analysis using the 177 DEGs, about 20 principal components were required to achieve the best performance (Figure 5E). For the selected DEP cases, however, only three principal components were required to obtain the best performance, which was the same accuracy as given by all components of PCA using DEPs. This also suggests that DEP-based features have meaningful discriminant information with respect to the HIV-1 stages.

\section{Discovery of HIV-1 stage-specific network modules using DEPs}

From the selected DEPs, we discovered HIV-1 stagespecific network modules. In this analysis, we also included genes that directly interact with the genes in the DEPs to extend the genes in DEPs. The extended network had 3545 nodes representing genes, with 50 739 edges between nodes denoting PPIs including DEPs. Using the extended network and an MCODE method (Methods), we identified five HIV-1-related network modules across the HIV-1 stages (Figure 6). Their network scores were 51.8, 45.0, 28.5, 14.0, and 11.0, respectively. Genes and interactions that composed a network module were predefined in the module-searching phase, but each model of a specific HIV-1 infection stage had its own representative co-expression scores and expression levels. In network module 5, for example, the coexpression score between $\mathrm{CDC} 7$ and MCM3 was negative only in the Uninfected stage. Additionally, the interactions between $\mathrm{CDC7}$ and MCM4 had identical signs for the co-expression score in Uninfected and Chronic stages or in Acute and Non-progressive stages, respectively. In GO enrichment analysis (Methods), all identified network modules were related to DNA or RNA metabolisms. More specifically, the main functional categories of the biological process of network modules 1 and 3 were "translation," including "translational elongation," "translation," and "gene expression." Module 2 was related to "RNA splicing," including "nuclear mRNA splicing," "RNA splicing via transesterification reactions," and "mRNA processing." Module 4 was related to "mRNA, RNA, and nucleic acid transport," and module 5 was associated with "DNA metabolism," including "DNA replication" and "DNA metabolic process." The HIV-related network modules revealed by network analysis using DEPs correspond with the results of some earlier studies (see Discussion for detailed explanation). The proposed DEP-based method, therefore, complemented the DEG-based approach in the microarray expression analysis of HIV-1 infection.

\section{Discussion}

The purpose of this study was to develop a novel microarray data analysis method to discover the stage-specific protein pairs in HIV-1 infection. The developed novel method focuses on the expression co-changing patterns between interacting protein pairs rather than on expression levels of individual genes. Note that we here only considered known PPIs that contact physically or chemically in selecting DEPs rather than all possible pairs among all detected genes in microarray; both because expressions noisy and because physically or chemically contacting pairs can share biological function and thus their biological meaning can be easily interpreted. Strength of our method comparing existing correlationbased method is that it can capture a genetic signature for a single sample. Even though one or more samples are used for selecting representative expression level, our method can identify a genetic signature for a new single sample by comparing with known representative expression level if those levels were already known by previous study.

With this method, 100 DEPs were selected for the discriminant features of HIV-1 stages. A comparison between DEPs and DEGs revealed that DEPs more powerfully classified the ambiguous stages of HIV-1. This means that DEPs can provide additional information not included in DEGs. As shown in Figure 2A, for example, the HIV-1-related proteins HNRNPM (600.8 under Acute and 595.0 under Chronic) and DHX9 (1477.7 under Acute and 1485.7 under Chronic) had similar expression levels between Acute and Chronic stages even though the variations within individual stages were relatively large (i.e., the expression level of DHX9 ranged from 420 to 1110 in the Chronic stage). Thus, the previous DEG-based approach missed both HNRNPM and DHX9 as the stage-specific genes for 


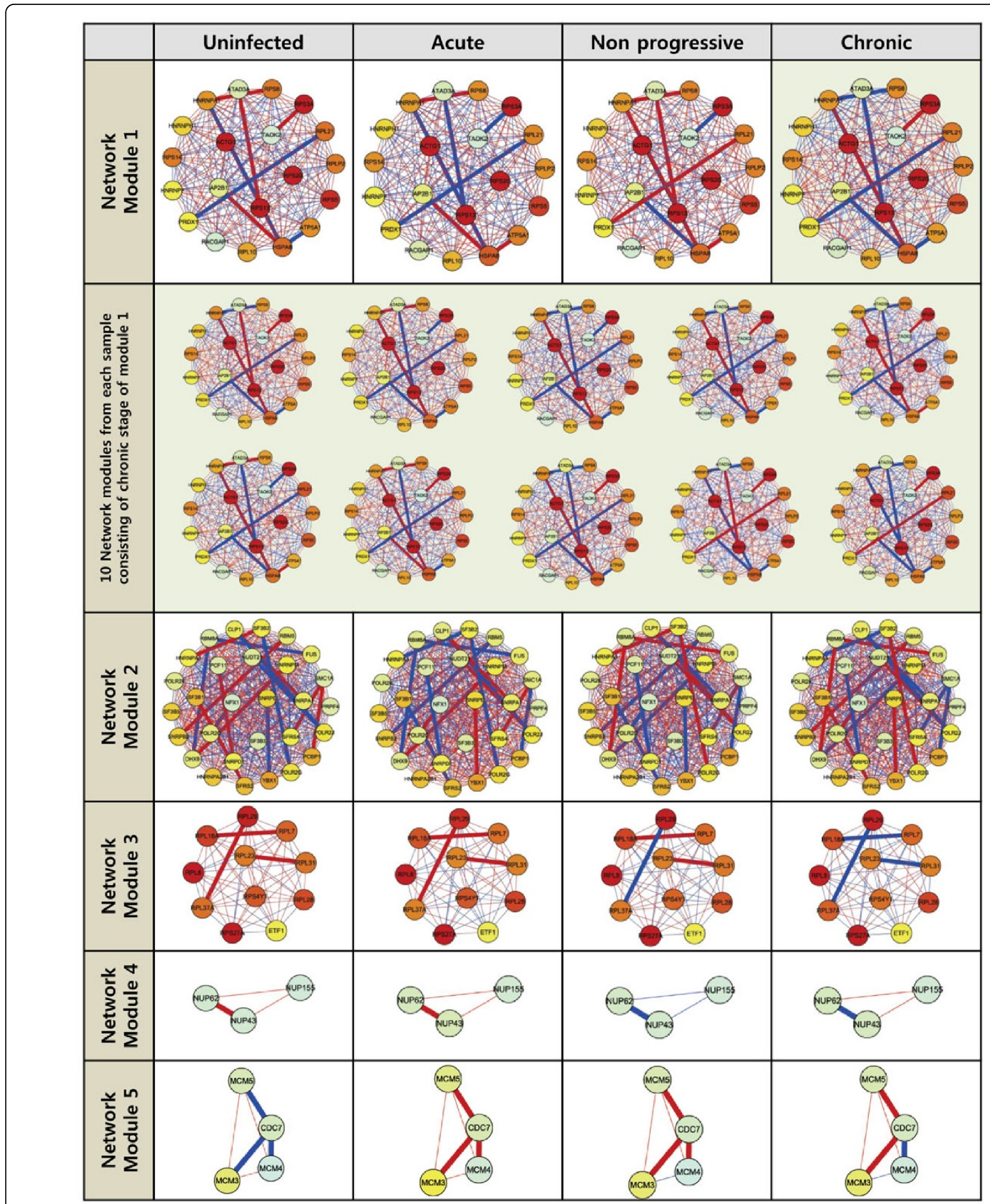

Figure 6 HIV-related network modules. Thin lines represent PPI, and thick lines denote DEPs. Nodes indicate protein, and node color denotes expression level. Blue, yellow, and red represent increasingly higher expressions of genes. The color of the edge represents the sign of the coexpressed score. If the sign is positive, the edge color is red, and if it is negative, blue is used. Representative modules for each stage were created using the median value of the co-expressed score and expression level of samples in the same stage. Network module 1 of the Chronic stage was made from 10 network modules listed in the second row. 
HIV-1. However, if we consider an expression co-changing pattern, the HNRNPM and DHX9 pair selected a significant feature of the HIV-1 stages because the cochanging scores were consistently positive in Acute samples but consistently negative in Chronic samples. In this respect, the DEP-based approach could well discriminate all four stages of HIV-1. Moreover, DEPs were enriched in more HIV-related GO terms, such as "apoptosis", which is strongly associated with the spectrum of the progression of HIV infection. Additionally, there is distinct difference between the DEP-based approach and previous correlation-based network analyses $[7,8]$. The biggest difference is that the DEP-based approach generates a distinct feature set with only one sample, whereas a correlation-based network approach finds a network feature with groups of samples under a specific condition. Thus, it is difficult to capture the characteristics of individual samples using the previous correlation-based network analyses. If there is a problem in predicting an unknown or new sample and if DEG-based analysis is unclear, then the DEP-based approach might be applicable.

The HIV-related network modules revealed by network analysis using DEPs correspond with the results of some earlier studies. Heterogeneous nuclear ribonucleoproteins (hnRNPs; complexes of RNA and protein) in modules 1 and 2 are known as HIV protein-synthesis modulators [27]. In module 2, SF3B2 (splicing factor 3b, subunit 2, $145 \mathrm{kDa}$ ) modulates viral proliferation of HIV through interaction with Vpr (Viral Protein R) [28]. SFRS2 (serine/arginine-rich splicing factor 2) influences the use of the HIV-1 splicing site [29]. SNRPE (small nuclear ribonucleoprotein polypeptide $\mathrm{E}$ ), one of the transcription elongation complexes, assembles with HIV Tat [30]. DHX9 (DEAH (Asp-Glu-Ala-His) box polypeptide 9) affects the expression of HIV-1 [31]. Furthermore, there is an association between PCF11 (PCF11, cleavage and polyadenylation factor subunit, homolog) and HIV-1 transcription[32]. PCBP1 (poly $(\mathrm{rC})$ binding protein 1) and YBX1 (Y-box binding protein 1) interact with Rev protein, a key regulator of HIV-1 gene expression [33]. NUP62 (nucleoporin $62 \mathrm{kDa}$ ) is related to Rev-mediated viral RNA export by interacting with eIF5A (eukaryotic translation-initiation factor 5A) [34]. In contrast to NUP62, NUP155 (nucleoporin $155 \mathrm{kDa}$ ) is associated with the import of HIV DNA [35]. All of these genes were included in DEPs but not in DEGs. From the module analysis with DEPs, it seems that changes in DNA and RNA metabolism are crucial in the clinical manifestations of HIV infection, and DEPs and HIV-related network modules might have the potential to assist in the elucidation of the pathogenesis of HIV-1 infection at the genomic and proteomic levels. However, further studies to seek biological confirmation are imperative to clarify the detailed roles of DEPs in specific HIV-1 stages.

\section{Conclusions}

We present a novel microarray data analysis method based on DEP by focusing on the expression co-changing patterns between interaction pairs. The DEP based algorithm was more powerful in classifying the ambiguous stages of HIV-1 and revealed the HIV-1 stage-specific network modules. The DEP-based method might contribute to complementation of existing DEG-based analyses.

\section{Acknowledgements}

This work was supported by the National Research Foundation of Korea (NRF) grant funded by the Korea government (MEST) (No.2010-0022887 and 2011-0018258). This research was also support by a grant (10182KFDA9922301) from Korea Food \& Drug Administration in 2011. H.S. was supported by the Priority Research Centers Program through the National Research Foundation of Korea (NRF) funded by the Ministry of Education, Science and Technology (2010-0028294).

This article has been published as part of BMC Systems Biology Volume 5 Supplement 2, 2011: 22nd International Conference on Genome Informatics: Systems Biology. The full contents of the supplement are available online at http://www.biomedcentral.com/1752-0509/5?issue=S2.

\section{Author details}

${ }^{1}$ Department of Biomedical Informatics, Ajou University School of Medicine, Suwon 443-749, Korea. ${ }^{2}$ Department of Anatomy, Ajou University School of Medicine, Suwon 443-749, Korea. ${ }^{3}$ Departments of Medicine and

Bioengineering, University of California San Diego, La Jolla, California 92093, USA.

\section{Authors' contributions}

Yoon, Lee contributed to the study conception and design. Yoon, Kim and Lee contributed to acquisition of data, analysis and interpretation of data. Yoon, Kim and Lee were responsible for drafting the manuscript. All authors have involved in revising the manuscript and have given final approval of the version to be published.

\section{Competing interests}

The authors have no conflicts of interest to declare.

Published: 14 December 2011

\section{References}

1. Pantaleo G, Graziosi C, Fauci AS: New concepts in the immunopathogenesis of human immunodeficiency virus infection. $N$ Engl J Med 1993, 328(5):327-335.

2. Chu C, Selwyn PA: Diagnosis and initial management of acute HIV infection. Am Fam Physician 2010, 81(10):1239-1244.

3. Grossman Z, Meier-Schellersheim M, Paul WE, Picker LJ: Pathogenesis of HIV infection: what the virus spares is as important as what it destroys. Nat Med 2006, 12(3):289-295.

4. Mothe B, Ibarrondo J, Llano A, Brander C: Virological, immune and host genetics markers in the control of HIV infection. Dis Markers 2009, 27(3):105-120.

5. Hyrcza MD, Kovacs C, Loutfy M, Halpenny R, Heisler L, Yang S, Wilkins O, Ostrowski M, Der SD: Distinct transcriptional profiles in ex vivo CD4+ and CD8+ T cells are established early in human immunodeficiency virus type 1 infection and are characterized by a chronic interferon response as well as extensive transcriptional changes in CD8+ T cells. J Virol 2007, 81(7):3477-3486

6. Li Q, Smith AJ, Schacker TW, Carlis JV, Duan L, Reilly CS, Haase AT: Microarray analysis of lymphatic tissue reveals stage-specific, gene 
expression signatures in HIV-1 infection. J Immunol 2009, 183(3):1975-1982.

7. Lin CC, Hsiang JT, Wu CY, Oyang YJ, Juan HF, Huang HC: Dynamic functional modules in co-expressed protein interaction networks of dilated cardiomyopathy. BMC Syst Biol 2010, 4:138.

8. Ma S, Shi M, Li Y, Yi D, Shia BC: Incorporating gene co-expression network in identification of cancer prognosis markers. BMC Bioinformatics 2010, 11:271.

9. Charbonnier S, Gallego O, Gavin AC: The social network of a cell: recent advances in interactome mapping. Biotechnol Annu Rev 2008, 14:1-28.

10. Lee K, Chuang HY, Beyer A, Sung MK, Huh WK, Lee B, Ideker T: Protein networks markedly improve prediction of subcellular localization in multiple eukaryotic species. Nucleic Acids Res 2008, 36(20):e136.

11. Salwinski L, Miller CS, Smith AJ, Pettit FK, Bowie JU, Eisenberg D: The Database of Interacting Proteins: 2004 update. Nucleic Acids Res 2004, 32(Database issue):D449-451.

12. Bader GD, Donaldson I, Wolting C, Ouellette BF, Pawson T, Hogue CW: BIND-The Biomolecular Interaction Network Database. Nucleic Acids Res 2001, 29(1):242-245.

13. Keshava Prasad TS, Goel R, Kandasamy K, Keerthikumar S, Kumar S, Mathivanan S, Telikicherla D, Raju R, Shafreen B, Venugopal A, et al: Human Protein Reference Database-2009 update. Nucleic Acids Res 2009, 37(Database issue):D767-772

14. Joshi-Tope G, Gillespie M, Vastrik I, D'Eustachio P, Schmidt E, de Bono B, Jassal B, Gopinath GR, Wu GR, Matthews L, et al: Reactome: a knowledgebase of biological pathways. Nucleic Acids Res 2005, 33(Database issue):D428-432

15. Rual JF, Venkatesan K, Hao T, Hirozane-Kishikawa T, Dricot A, Li N, Berriz GF, Gibbons FD, Dreze M, Ayivi-Guedehoussou N, et al: Towards a proteomescale map of the human protein-protein interaction network. Nature 2005, 437(7062):1173-1178.

16. Stelzl U, Worm U, Lalowski M, Haenig C, Brembeck FH, Goehler H, Stroedicke M, Zenkner M, Schoenherr A, Koeppen S, et al: A human protein-protein interaction network: a resource for annotating the proteome. Cell 2005, 122(6):957-968.

17. Ewing RM, Chu P, Elisma F, Li H, Taylor P, Climie S, McBroom-Cerajewski L, Robinson MD, O'Connor $L$, Li M, et al: Large-scale mapping of human protein-protein interactions by mass spectrometry. Mol Syst Biol 2007, 3:89.

18. Ramani AK, Bunescu RC, Mooney RJ, Marcotte EM: Consolidating the set of known human protein-protein interactions in preparation for large-scale mapping of the human interactome. Genome Biol 2005, 6(5):R40

19. Saeed Al, Bhagabati NK, Braisted JC, Liang W, Sharov V, Howe EA, Li J, Thiagarajan M, White JA, Quackenbush J: TM4 microarray software suite. Methods Enzymol 2006, 411:134-193.

20. Hall Mark, F E, Holmes Geoffrey, Pfahringer Bernhard, Reutemann Peter, Witten Hlan: The WEKA Data Mining Software: An Update. SIGKDD Explorations 2009, 11(1):10-18

21. Xu H, Lemischka IR, Ma'ayan A: SVM classifier to predict genes important for self-renewal and pluripotency of mouse embryonic stem cells. BMC Syst Biol 2010, 4(1):173.

22. Cline MS, Smoot M, Cerami E, Kuchinsky A, Landys N, Workman C, Christmas R, Avila-Campilo I, Creech M, Gross B, et al: Integration of biological networks and gene expression data using Cytoscape. Nat Protoc 2007, 2(10):2366-2382.

23. Bader $\mathrm{GD}$, Hogue $\mathrm{CW}$ : An automated method for finding molecular complexes in large protein interaction networks. BMC Bioinformatics 2003, 4:2.

24. Huang da W, Sherman BT, Lempicki RA: Systematic and integrative analysis of large gene lists using DAVID bioinformatics resources. Nat Protoc 2009, 4(1):44-57.

25. Maere S, Heymans K, Kuiper M: BiNGO: a Cytoscape plugin to assess overrepresentation of gene ontology categories in biological networks. Bioinformatics 2005, 21(16):3448-3449.

26. Alimonti JB, Ball TB, Fowke KR: Mechanisms of CD4+ T lymphocyte cell death in human immunodeficiency virus infection and AIDS. J Gen Virol 2003, 84(Pt 7):1649-1661.

27. Bolinger $C$, Boris-Lawrie $K$ : Mechanisms employed by retroviruses to exploit host factors for translational control of a complicated proteome. Retrovirology 2009, 6:8.
28. Hashizume C, Kuramitsu M, Zhang X, Kurosawa T, Kamata M, Aida Y Human immunodeficiency virus type $1 \mathrm{Vpr}$ interacts with spliceosomal protein SAP145 to mediate cellular pre-mRNA splicing inhibition. Microbes Infect 2007, 9(4):490-497.

29. Ropers D, Ayadi L, Gattoni R, Jacquenet S, Damier L, Branlant C, Stevenin J: Differential effects of the SR proteins 9G8, SC35, ASF/SF2, and SRp40 on the utilization of the $\mathrm{A} 1$ to $\mathrm{A} 5$ splicing sites of HIV-1 RNA. J Biol Chem 2004, 279(29):29963-29973.

30. Sobhian B, Laguette N, Yatim A, Nakamura M, Levy Y, Kiernan R, Benkirane M: HIV-1 Tat assembles a multifunctional transcription elongation complex and stably associates with the 7SK snRNP. Mol Cell 2010, 38(3):439-451.

31. Bolinger C, Sharma A, Singh D, Yu L, Boris-Lawrie K: RNA helicase A modulates translation of HIV-1 and infectivity of progeny virions. Nucleic Acids Res 2010, 38(5):1686-1696.

32. Klatt A, Zhang Z, Kalantari P, Hankey PA, Gilmour DS, Henderson AJ: The receptor tyrosine kinase RON represses HIV-1 transcription by targeting RNA polymerase II processivity. J Immunol 2008, 180(3):1670-1677.

33. Hadian K, Vincendeau M, Mausbacher N, Nagel D, Hauck SM, Ueffing M, Loyter A, Werner T, Wolff H, Brack-Werner R: Identification of a heterogeneous nuclear ribonucleoprotein-recognition region in the HIV Rev protein. J Biol Chem 2009, 284(48):33384-33391.

34. Hofmann W, Reichart B, Ewald A, Muller E, Schmitt I, Stauber RH, Lottspeich F, Jockusch BM, Scheer U, Hauber J, et al: Cofactor requirements for nuclear export of Rev response element (RRE)- and constitutive transport element (CTE)-containing retroviral RNAs. An unexpected role for actin. J Cell Biol 2001, 152(5):895-910.

35. Lee K, Ambrose Z, Martin TD, Oztop I, Mulky A, Julias JG, Vandegraaff N, Baumann JG, Wang R, Yuen W, et al: Flexible use of nuclear import pathways by HIV-1. Cell Host Microbe 2010, 7(3):221-233.

doi:10.1186/1752-0509-5-S2-S1

Cite this article as: Yoon et al:: Differentially co-expressed interacting protein pairs discriminate samples under distinct stages of HIV type 1 infection. BMC Systems Biology 2011 5(Suppl 2):S1.

\section{Submit your next manuscript to BioMed Central and take full advantage of:}

- Convenient online submission

- Thorough peer review

- No space constraints or color figure charges

- Immediate publication on acceptance

- Inclusion in PubMed, CAS, Scopus and Google Scholar

- Research which is freely available for redistribution 